International Journal of Energy for a Clean Environment, 13(1-4), vii-xiii (2012)

\title{
EMERGING PROBLEMS OF NUCLEAR POWER PLANT'S SAFETY AND EFFICIENCY IMPROVEMENT
}

\author{
Igor I. Sviridenko
}

Sevastopol State Technical University, Sevastopol, Ukraine

The article presents main directions of scientific research of the Annual International Scientific and Practical Conference "Safety, Efficiency, Lifetime of Nuclear Power Installations" ("ICNPE") participants, which was organized in Sevastopol, Ukraine. This year was already the 10th conference dedicated to safety improvement of the stationary and transport reactor installations, efficiency of operation and lifetime management; the continuous growth of number of participants and companies, delegating their representatives to the conference, indicate growing interest in the ICNPE conference from the nuclear industry. This is evidence of the expediency of such an international conference and the possibility of information exchange within the international nuclear society.

This special edition of the "International Journal of Energy for a Clean Environment" is prepared on the basis of materials of International Scientific and Practical Conference "Safety, Efficiency, Lifetime of Nuclear Power Installations," which annually takes place in Sevastopol, Ukraine with support of the State Nuclear Regulatory Inspection of Ukraine.

The main topic of ICNPE is discussions about new challenges for the nuclear industry, related to the safety, reliability, and efficiency improvement of nuclear power plant (NPP) operation, approbation of research results, and innovative developments in the field of nuclear machinery, establishing scientific and commercial relations.

Traditional subjects of ICNPE are the following:

- Safety, reliability, and efficiency improvement of operation of nuclear installations.

- Prospective nuclear installations and NPPs of future generations.

- Modern methods for diagnostic, in-service inspection, and maintenance of NPP components.

- Aging management, long-term operation, decommissioning of NPPs.

- Fuel handling. Radwaste storage and transportation.

- State-of-the-art analytical modeling of processes in nuclear installations.

- Training of NPP personnel.

Scientists and nuclear experts from Ukraine, Russia, Germany, Czech Republic, Belorussia, and Lithuania are taking part in the conference.

The last conferences took place in the post-Fukushima period. This was the first time in the world of nuclear practice that such a severe accident was caused by 
natural phenomena, and after an earthquake and tsunami both normal and emergency power sources were lost, which led to the loss of residual heat sink from reactors and spent fuel pools. Overheating, evaporation, and consequent loss of primary and spent fuel pool inventory led to fuel overheating and failure, and in reactor cores - to core melt. Accumulation of hydrogen generated due to steamzirconium reaction led to explosions and fires.

Taking into account lessons learned from the accident, the biggest interest of participants was attracted by the results of the latest safety analysis reports of operating NPPs and presentations containing proposals and recommendations regarding prevention of severe accidents under conditions, similar to the FukushimaDaiichi event.

In the ICNPE framework there were "round table talks" with the topic: "NPP Safety improvement issues related to extreme external events. Lessons learned from Fukushima." Results of risk assessments conducted at NPPs (stress tests) have been discussed during the round table talks corresponding to obligations taken according to the Nuclear Safety Convention of 2011.

The main task of the conducted "stress tests" was assessment of the influence of possible extreme natural events: earthquakes, flooding, fires, tornadoes, extreme high/low temperatures, extreme rain/snow, strong wind, and also the influence of combination of extreme external events on NPPs. Special attention has been addressed to the assessment of a NPP under total power loss and/or loss of residual heat sink conditions, and also possibilities to manage severe accidents.

The round table talks participants underlined that, even though such an extreme external event as earthquake and tsunami like which happened at the FukushimaDaiichi NPP is very unlikely for the majority of NPPs, the technical means, providing long-term heat sink under total loss of all power sources, at the majority of NPPs which are currently in operation are not available. Consequently, to mitigate the negative impact of extreme external events potentially leading to the complete long-term loss of all normal and emergency power sources, the availability of mobile power supply systems and means of forced water circulation is of key importance. In addition to that, development and introduction of passive residual heat removal systems from the reactor core and spent fuel pool and passive molten core retention systems are also important.

ICNPE participants highlighted the importance of combination of active and passive safety systems, performing critical safety functions in full scope that practically excludes the impact of accidents related to complete loss of all power and loss of residual heat sink. Examples of such approach are evolutionary designs of NPPs with WWER reactors ("Atomenergoproekt," Russia): Kudankulam NPP (India), Novovoronezh-2, Leningrad-2, Baltic NPPs (Russia), Akkuyu NPP (Turkey).

There is another, more revolutionary approach in solving this issue, considering complete replacement of active safety systems by passive safety systems, as in AP1000 design (Westinghouse, USA). 
ICNPE participants proposed to establish an international crisis center of mitigation of consequences of possible severe nuclear accidents based, possibly, in Ukraine - a country of the Chernobyl accident. Experts with experience in mitigation of severe accidents at Chernobyl NPP, Three Mile Island, and FukushimaDaiichi could be invited to the Center.

One of the most discussed topics at ICNPE was the area of application of stateof-the-art methods of analytical modeling of emergency transients in nuclear power installations. The topical works are development and verification of safety assessment codes for nuclear installations, development of a bank of experimental data for verification of neutron-physical and thermo-hydraulical codes, and also implementation of uncertainty assessment methodology. Following the results of analytical modeling of beyond-design emergency transients, and also following the results of analytical research, severe accident management guidelines for specific NPPs are being developed. Enhancement of the modern software and analytical codes for modeling of processes in nuclear installations remains one of the top priority activities.

Special attention is paid to experimental substantiation of the introduction of new passive safety systems and systems for severe accident management with identification of all phenomena with possible impact on efficiency of the developed systems.

Innovative developments, providing safety improvement of NPPs during emergency transients, are appealing and of great interest. One of those is the development of passive safety systems based on two-phase thermosiphons. These are passive systems for residual heat removal (PRHRS) from reactor, PRHRS for spent fuel pool, PRHRS for containment, means of passive thermal protection for secondary piping's containment penetrations.

Two-phase thermosiphons are autonomous closed-cycle heat transfer systems, with high efficiency, reliability, and simple design. Application of the heat exchangers based on the two-phase thermosiphons allows efficient and reliable heat transfer from heat source to the final heat repository and, simultaneously, provides an additional safety barrier protecting people and the environment from ionizing radiation.

Two-phase thermosiphons were proposed as a basis for the development of passive cooldown system for the WWER pressurizer, which, along with thermal siphon-based reactor PRHRS, will provide conditions where reactor subcriticality is maintained by boron injection from emergency core cooling system hydro accumulators (ECCS HA), without the need to increase the efficiency of reactor protection control rods and the introduction of additional systems for fast boron injection. Such a complex system can prevent core damage in the case of complete long-term blackout. In addition to that, it provides significantly (in order of magnitude) increased time till core failure for cases with coincident small loss-ofcoolant accidents (LOCAs). Analyses show little sensitivity of thermosiphon-based

Volume 13, Numbers 1-4, 2012 
PRHRS in the reactor vessel to the presence of noncondensable gases in the primary circuit.

Practical interest was also attracted by an innovative containment cooldown system for cases of primary or secondary LOCA, based on the use of jet spray coolers. The system works by generation of sprayed water flow, capable of ejecting thousands of cubic meters of air-steam mixture per cubic meter of working liquid, decreasing the steam temperature to saturation line and its consequent condensation. The approach allows efficient limitation of containment pressure growth during LOCA accidents by local condensation of steam in a separated volume of a jet cooler with further draining of the condensed and cooling sprinkling media through drainage pipes into a tank collector.

The main benefit of the proposed approach is the creation of conditions where the containment pressure does not exceed the containment localization pressure setpoint. Actuation of the containment spray pump to supply cooling media for jet coolers provides an early decrease of containment pressure and prevents water injection through upper sprinklers. As a result, primary equipment will not be directly sprayed by boron solution. Moreover, after connection of the safety injection system to operation from the containment sump, the equipment will not be sprayed with contaminated water, preventing the unnecessary spread of contamination. Specifically, the conference highlights that introduction of the jet coolers prevents events similar to what happened at Rovno NPP in September 2009.

In addition to that, there is a possibility of development of a jet-cooler-based passive containment cooldown system. The operation principle of such a system is based on water supply to jet-cooler's sprinklers from passive nitrogen-pressurized hydro accumulators.

A promising topic for WWER 1000 safety improvement under emergency cooldown in the case of LOCA accident is introduction of a possibility to throttle high-pressure (HP) ECCS and low-pressure (LP) ECCS flow. Control of HP ECCS flow during primary-to-secondary LOCA allows preventing of primary coolant release to the environment through steam dump valves. In the case of a primary LOCA, there is a possibility to keep in operation two LP ECCS trains one for cooldown and another one to maintain primary coolant inventory. For WWER 1000 "Small Series" Units it provides the efficient use of boron solution in the case of small LOCAs. That also prevents overcooling of the primary side under high pressure (thermal hammers, thermal shock).

Traditionally, ICNPE considers proposals regarding introduction of state-of-theart technology, allowing efficient solutions toward increase of efficiency and reliability of the main equipment of WWER 1000 NPPs. Especial important is the topic of degradation of PGW-1000 Steam Generators primary collector's welds. This issue is extremely sensitive because correction of identified faults during operation requires significant time, finances, and high dose load for the personnel involved. New technology, proposed during the conference, allows the decreasing 
propagation of existing micro-cracks and prevention of initiation of similar faults in intact areas.

To increase the operational safety of NPPs, and to increase efficiency by lowering operational costs, a set of recommendations has been developed in modernization or replacement of outdated and obsolete systems and components. One of the promising subjects is replacement of ventilator-equipped safety system's cooling towers by jet-vortex cooling towers, with higher reliability and replacement of spray ponds by jet-vortex cooling towers. Jet-vortex cooling towers are not sensitive to tornadoes, they can work using existing pumps and piping, and they are able to improve cooldown by $3-5^{\circ} \mathrm{C}$, decreasing water loss to evaporation till $0.8 \%$.

An important issue for NPPs with WWER reactors is maintaining design power level. Recently, using analytical modeling, experts performed analysis of efficiency of engineering solutions for upgrading cooling capabilities of the Tashlyk cooling pond of the South Ukraine NPP. A hydrodynamic model of the cooling capabilities has been composed and the results are in good agreement with corresponding fluctuations of water temperatures in the cooling pond. The results revealed that, having two power units in operation, comfortable temperature in the cooling pond can be reached by construction of a flow control wall. When three power units are in operation and a part of the cooling pond is isolated by a pump-storage plant's dam, maintaining the operational temperature range may require introduction of spray ponds. The results have also shown the possibility of online forecast of cooling pond temperature, based on meteorological modeling of weather forecast and hydrothermal-dynamic modeling of the cooling pond.

Suppliers of NPP equipment are also taking an active part in ICNPE. These are mostly producers of diagnostic equipment. This is a sign of increased interest of the suppliers to issues related to implementation of new state-of-the-art diagnostic methods and instruments which has a positive impact on reliable operation, maintenance cost reduction, aging management, and lifetime extension.

The conference mentioned that many operating companies do not have a sufficient range of state-of-the-art diagnostic equipment. For example, WWER 1000 plants in their design do not have full range of means allowing monitoring plant conditions along and after severe and beyond-design basis accidents. In lieu of this, increased interest was addressed to PAM System (Post-Accident Monitoring), designed to provide the operator with reliable and timely information about WWER Unit and environment during and after an accident with "harsh" conditions. The PAMS has been designed taking into account safety improvement requirements, recommendations of IAEA, and other standards.

Reports addressing environmental aspects of nuclear industry are especially popular at ICNPE. The great interest is in present innovative technologies of nuclear and radiochemical industry sewerage water purification, and also sanitation of natural water sources from radio isotopes. The liquid radwaste treatment method proposed at the conference is based on the use of natural mineral sorbents with

Volume 13, Numbers 1-4, 2012 
high specific surface, achieved due to partial chemical, mechanical, hydro-dynamical, electro-dynamical, plasma, or cavitational fragmentation of the minerals, partially till nanosize. The method allows minimization of costs of the liquid radwastes treatment by use of widely available natural minerals as the basis for sorbent production. The authors practically proved the possibility of liquid radwaste treatment, even with high salt background and containing impurities in the form of organic and mineral oils and in the form of surfactants.

One article presented in the current IJECE issue presents in detail the method of evaporation of vat residue, conducted in the presence of oxidant aero-ions with preliminary added nanostructured sorbent, produced from natural aluminosilicate. The distinguishing feature of the nanostructured sorbent is that, except for absorption of the radio nuclides, it has the ability to absorb heavy metals, salts, borates, and other toxic substances, and also to serve as a binding element. Evaporation results in formation of the sorption-crystalline precipitate and condensate, which can be used for in-house purposes of the NPP. Sorption-crystalline precipitate is an intermediate for high chemically resistant glassy substance, received by melting the sludge in an induction furnace. The proposed method allows the simplification of radwaste treatment technology without formation of the salt melt, transfer salt to melted glass form, simplify handling, storage, and solidification of the bottoms. Prototype testing of the nanosorbent confirms the high efficiency of the method and its potential ability for wide application in liquid radioactive waste processing at NPPs.

Following the Fukushima-Daiichi accident, where a large amount of radioactivity was released to the atmosphere and there was direct release of nuclides to coastal waters, there arose the problem of realistic assessment of possible consequences of the releases.

On the request of international organizations and addressing public interest in getting realistic assessment of possible consequences of the severe accident, existing multiplatform revision of the Java language real-time on-line decision support (JRODOS) system was quickly adapted for the Fukushima-Daiichi site. The adaptation has been performed by experts from Karlsruhe Technological Institute (KIT) in tight collaboration with Ukrainian experts. Assessment of radioactive contamination was received on the basis of Japan Agency of Nuclear and Industrial Safety (NISA) and results of diagnostic assessment of the weather forcast model (WRF) meteorological model, preliminary verified with meteorological data from Tokyo and Fukushima Airports. Diagnostic calculations of RODOS satisfactorily represent radioactivity map in the NPP area. The experience gained in setting up the JRODOS system for operational application to the Japanese NPP will be further used by the developers to continuously improve the system and to adopt their work for other countries.

One environment-related activity in the nuclear industry is organization and conduct of environmental monitoring in the NPP area. On the example of Europe's largest Zaporozhye NPP, the features of hydrochemical changes of surface waters 
of the adjacent area were analyzed. The structure of computerized environmental monitoring system of heavy metals content in the NPP cooling pond's water was presented. An analytical tool for assessment and forecast of the water quality in different operational modes of the cooling pond, cooling towers, and jet spray ponds was presented. Visualization of distribution of depth in the cooling pond of a NPP was presented. A comparative analysis of the experimental and predicted data of water quality on the content of their copper has been made.

A hot topic is the methodology of strategic planning for the decommissioning of nuclear facilities. On the example of implementation of decommissioning of USSR's nuclear Navy facilities, the complexity and interconnectedness of problems of preparation and decommissioning and subsequent disposal of nuclear facilities has been demonstrated. ICNPE points out the importance of complex preparation of decommissioning of NPPs when their designed lifetime expires.

Taking into account Human Factor's impact on NPP's safety, man-machine interface issues, consistency of procedures, form and content of informational support, alarms and level of the automation of processes for plant operators are also subject of analyses.

Results of scientific and research works presented in articles of this specific issue are playing a great role in selection and substantiation of technical decisions related to the safety of NPPs. Proposals and recommendations are addressed to design and operating companies working in the nuclear industry for consideration and application in practical activities related with safety and efficiency improvement of NPPs.

Volume 13, Numbers 1-4, 2012 